\title{
Association of blood glucose level and prognosis of inpatients with coexistent diabetes and COVID-19
}

\author{
Weiwei Duan $\mathbb{1}^{1,2,3,4} \cdot$ Liangyu $\mathrm{Li}^{1,2} \cdot$ Xuesong $\mathrm{Li}^{5} \cdot$ Mengyan $\mathrm{Zhu}^{1,2} \cdot$ Lingxiang $\mathrm{Wu}^{1,2} \cdot$ Wei $\mathrm{Wu}^{1,2} \cdot \mathrm{Kening} \mathrm{Li}^{1,2} \cdot$

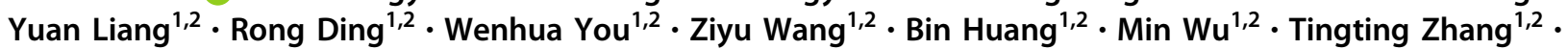 \\ Jie $\mathrm{Li}^{1,2} \cdot \mathrm{Yan}_{\mathrm{Li}^{1,2}}$. Jiaofang Shao, ${ }^{1,2}$ Changsong $\mathrm{Lin}^{1,2} \cdot$ Pengping $\mathrm{Li}^{1,2} \cdot$ Qianghu Wang ${ }^{1,2,3,4}$. Shukui Wang ${ }^{6}$. \\ Xinyi $\mathrm{Xia}^{7,8} \cdot \mathrm{Yu} \mathrm{Liu}^{9} \cdot$ Sali Lyu $\mathrm{Lu}^{1,2,3,4}$
}

Received: 10 August 2021 / Accepted: 19 October 2021 / Published online: 30 October 2021

(c) The Author(s), under exclusive licence to Springer Science+Business Media, LLC, part of Springer Nature 2021

\begin{abstract}
Type 2 diabetes (T2D) increases the risk of coronavirus disease (COVID-19). This study investigates the association between glucose control of COVID-19 patients with T2D in first 7 days after hospital admission and prognosis. A total of 252 infected inpatients with T2D in China were included. Well-controlled blood glucose was defined as stable fasting blood glucose (FBG) levels in the range of 3.9-7.8 mmol/L during first 7 days using indicators of average $\left(\mathrm{FBG}_{\mathrm{A}}\right)$, maximum $\left(\mathrm{FBG}_{\mathrm{M}}\right)$ or first-time $\left(\mathrm{FBG}_{1}\right) \mathrm{FBG}$ levels. The primary endpoint was admission to intensive care unit or death. Hazard ratio (HR) of poorly controlled glucose level group compared with well-controlled group were $4.96(P=0.021)$ for $\mathrm{FBG}_{\mathrm{M}}$ and $5.55(P=0.014)$ for $\mathrm{FBG}_{\mathrm{A}}$. Well-controlled blood glucose levels in first 7 days could improve the prognosis of COVID-19 inpatients with diabetes.
\end{abstract}

Keywords COVID-19 $\cdot$ Type 2 diabetes $\cdot$ Glucose control $\cdot$ Prognosis analysis

These authors contributed equally: Weiwei Duan, Liangyu Li, Xuesong Li, Mengyan Zhu

Supplementary information The online version contains supplementary material available at https://doi.org/10.1007/s12020021-02923-7.

Weiwei Duan

passion@njmu.edu.cn

$\triangle$ Xinyi Xia

xinyixia@nju.edu.cn

$\triangle$ Yu Liu

drliuyu@njmu.edu.cn

$\triangle$ Sali Lyu

lvsali@njmu.edu.cn

1 Research Center for Global Health, School of Public Health, Nanjing Medical University, Nanjing, China

2 Department of Bioinformatics, Nanjing Medical University, Nanjing, China

3 Collaborative Innovation Center for Cardiovascular Disease Translational Medicine, Nanjing Medical University, Nanjing, China
4 Jiangsu Key Lab of Cancer Biomarkers, Prevention and Treatment, Collaborative Innovation Center for Personalized Cancer Medicine, Nanjing Medical University, Nanjing, China

5 Key Laboratory of Cardiovascular and Cerebrovascular Medicine, School of Pharmacy, Nanjing Medical University, Nanjing, China

6 Department of Laboratory Medicine, Nanjing First Hospital, Nanjing Medical University, Nanjing, China

7 COVID-19 Research Center, Institute of Laboratory Medicine, Jinling Hospital, Nanjing University School of Medicine, Nanjing Clinical College of Southern Medical University, Nanjing, China

8 Joint Expert Group for COVID-19, Department of Laboratory Medicine \& Blood Transfusion, Wuhan Huoshenshan Hospital, Wuhan, Hubei 430100, China

9 Department of Endocrinology, Sir Run Run Hospital, Nanjing Medical University, Nanjing, China 


\section{Abbreviations}

COVID-19 Coronavirus disease 2019

SARS-CoV-2 severe acute respiratory syndrome coronavirus 2

ICU intensive care unit

HR hazard ratio

95\% CI $\quad 95 \%$ confidence interval

FBG fasting blood glucose

CVD cardiovascular and cerebrovascular diseases

IQR interquartile range

COPD chronic obstructive pulmonary disease

CPT convalescent plasma transfusion

\section{Introduction}

The coronavirus pandemic, caused by a novel coronavirus, the severe acute respiratory syndrome coronavirus 2 (SARSCoV-2), has spread all over the world since Dec 2019 [1]. By the end of 2020, confirmed coronavirus cases surpassed 90 million globally according to reports by WHO.

Studies have suggested that most people affected by coronavirus disease 2019 (COVID-19) have comorbidities, the most prevalent of which are hypertension, diabetes, and cardiovascular disease [2-5]. Generally, about $10-20 \%$ of patients with COVID-19 had diabetes. Research suggests that the patients with diabetes were more susceptive to SARS-CoV-2 and subsequently had poor COVID-19 prognosis [6-8]. Thus, the patients with diabetes require more attention from the perspective of either prevention during pandemic or hospitalized treatment after infection. Glucose management is a universal topic for diabetes therapy. Hyperglycemia is detrimental to inflammation control and yields high risk of secondary infection and mortality [9]. Relationship between glucose level management and survival outcomes of general infected inpatients has been reported [10-12]. However, few studies described this association in infected inpatients with pre-existing diabetes. Some researchers have clarified the importance and provided insights for glucose control in patients with diabetes and COVID-19 [13-15].

In order to investigate the association between blood glucose control and prognosis of patients with diabetes and COVID-19, we retrospectively analyzed 206 inpatients diagnosed with type 2 diabetes (T2D) and lab-confirmed COVID-19 admitted to a designated isolation medical center in Wuhan Huoshenshan hospital from February 4th to March 30th 2020. We showed the poorly controlled fasting blood glucose (FBG) levels $(>7.8 \mathrm{mmol} / \mathrm{L})$ put the hospitalized patients with diabetes and COVID-19 at high risk of admission to intensive care unit (ICU) or in-hospital death. One group in China reported a large populationbased study also focusing on link between blood glucose level management and prognosis of patients with diabetes and COVID-19 [7]. Although relatively small sample size in this study, however, there is some significant difference between these two works in outcome selection, recommended level for FBG control, handing dynamic FBG. We also discover whether FBG at admission can predict the prognosis. We hope our findings can raise more concern about blood glucose management for patients with COVID19 and diabetes.

\section{Methods}

\section{Study population}

There were 3057 laboratory-confirmed COVID-19 cases admitted to Huoshenshan Hospital in Wuhan, China, from February 4th to March 30th 2020. This retrospective study included 1568 cases with highest grade being severe or critical during hospitalization [16]. The severity grade of COVID-19 patients was diagnosed based on the diagnostic and treatment guideline (Version 5-7) by the National Health Committee of China, and varied during hospitalization. We extracted demographic, clinical characteristics, laboratory findings and prognosis of inpatients from electronic medical records (EMR). Clinical outcomes were followed up to April 10th, 2020. A total of 252 of 1568 patients were defined as preexisting T2D patients based on selfreported T2D or drugs for glycemic control. We excluded the subjects without available FBG readings in first 7 days after admission $(n=30)$, those without FBG measurement before admitting to ICU $(n=5)$, and those with hypoglycemia $(\mathrm{BG}<3.9 \mathrm{mmol} / \mathrm{L})(n=3)$ or age beyond $19-85$ years $(n=8)$. Finally, 206 diabetes patients were remained in our final analysis. This study was approved by the Medical Ethical Committee of Wuhan Huoshenshan Hospital. The informed consent was waived by the ethics board of the hospital due to urgency need during this pandemic. All procedures were in accordance with the World Medical Association's Declaration of Helsinki.

\section{Blood glucose measurement and survival outcome}

FBG levels and time of blood sampling was extracted from EMR. The time and frequency of FBG examination varied within subjects depending on clinic need. We mainly concentrated on the FBG values in 7 days after admission. The arithmetic mean $\left(\mathrm{FBG}_{\mathrm{A}}\right)$, maximum $\left(\mathrm{FBG}_{\mathrm{M}}\right)$ and first-time $\left(\mathrm{FBG}_{1}\right)$ levels for dynamic measurement of FBG in each patient was calculated to represent average, worse and baseline (admission) glucose control, respectively. With the references from others, we selected $\leq 7.8 \mathrm{mmol} / \mathrm{L}$ as a criteria for well controlled FBG 


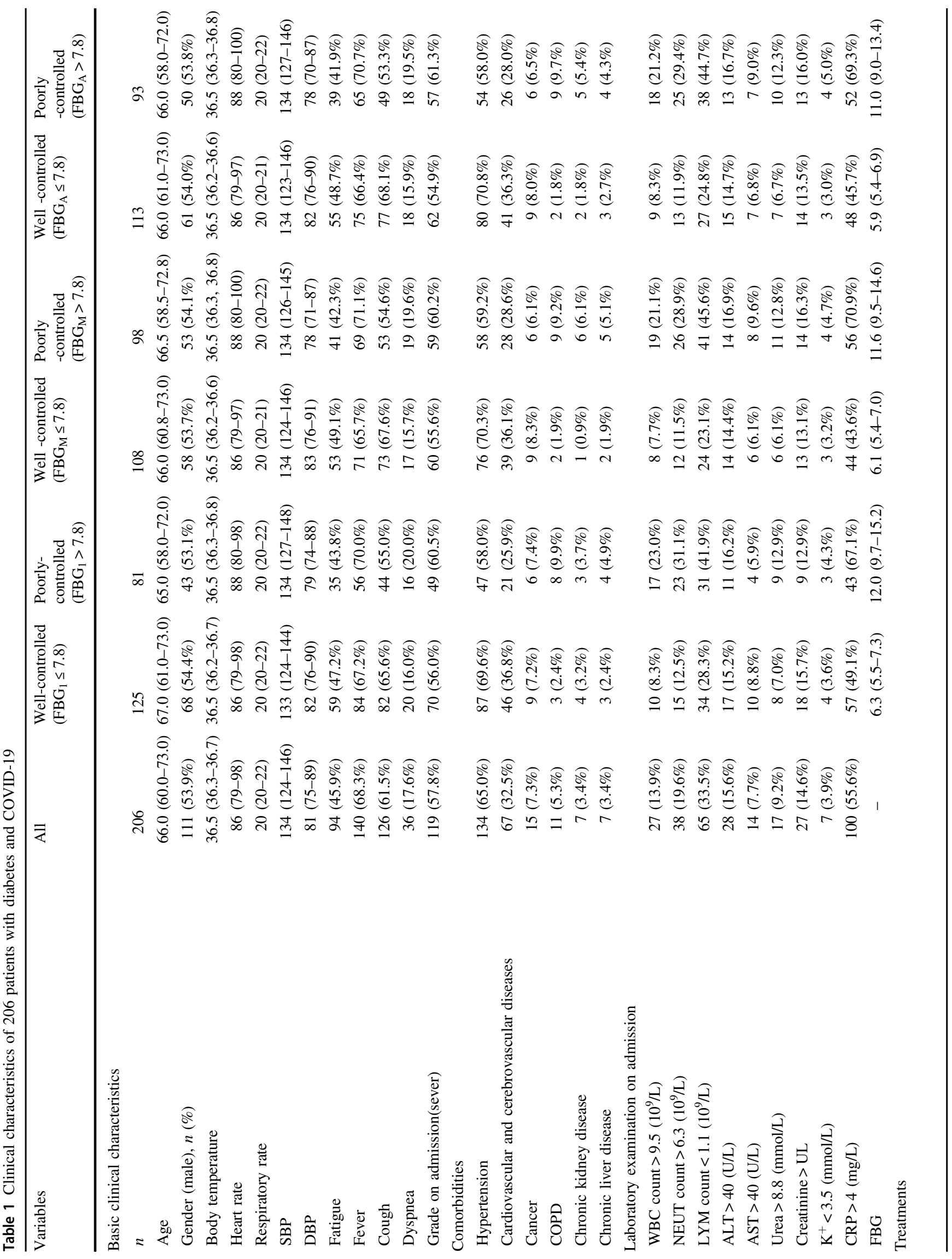


of inpatients $[9,13]$. We also used cutoff at $10.0 \mathrm{mmol} / \mathrm{L}$ for grouping as a sensitive analysis [7]. Although hemoglobin A1c (HbA1c) test is thought to be more accurate and reliable than FBG test, the small number of patients had HbA1c being tested. However, we also extracted available $\mathrm{HbA} 1 \mathrm{c}$ from only 33 patients to illustrate that FBG in 7 days show better consistency with HbA1c than FBG in 28 days used in others [7].

The outcomes included discharging from hospital, hospitalization, and death. We defined primary endpoint as admission to ICU or in-hospital death.

\section{Statistical analysis}

Continuous and categorical variables were presented as median (interquartile range, IQR) and $n(\%)$, respectively. Local polynomial regression was used to smooth the dynamic glucose change across days after admission. Pearson correlation coefficient (PCC) was used to evaluate the linear correlation between continuous variables. We employed Cox proportional hazard model to estimate the hazard ratio (HR) with 95\% confidence interval (CI) of FBG management (binary, well-controlled vs. poorly controlled status) surrogated by $\mathrm{FBG}_{\mathrm{A}}, \mathrm{FBG}_{\mathrm{M}}$ or $\mathrm{FBG}_{1}$ on survival outcome. Two models were built to evaluate the underlying confounding effects of various combinations of covariates. Model 1 was adjusted for age (continuous), gender (binary) and severity classification on admission (binary, non-severe vs. severe). Model 2 furtherly accounted for comorbidities including hypertension (binary), CVD (binary), COPD (binary) and combined other comorbidities (binary), treatments including antivirus drug used (binary), convalescent plasma transfusion (CPT) therapy (binary) and steroid used (binary). The combined variable was a binary indictor defined as patients with any of cancer, chronic kidney disease and chronic liver disease. We used the scaled Schoenfeld residuals to check the proportional hazard assumption in Cox regression [17]. Furthermore, potential nonlinear relationship between FBG and outcome was discovered by generating a restricted cubic spline term of FBG in Cox regression with specifying 3 to the number of knots.

We used R software (version 3.6.2) for all analyses. All statistical tests were two-sided and $P<0.05$ was considered to be statistically significant.

\section{Results}

In total, there were 206 patients with diabetes and COVID-19 included in this study with mean follow-up of 16.9 days. Sixteen patients reached the endpoint. The general clinical characteristics of the participants are shown in Table 1. Median age was 66 (IQR, 60-73) and 53.9\% of participants were male. 
(A) $\mathrm{FBG}_{1}$ group

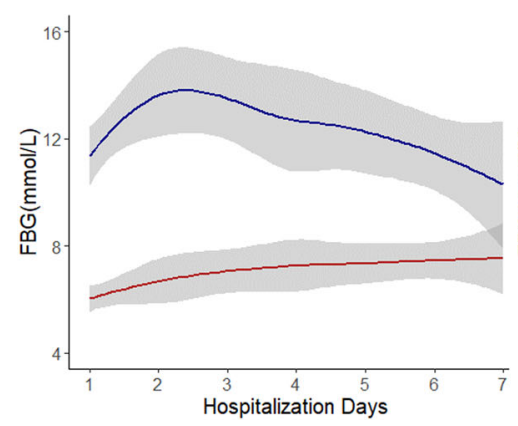

(B) $F B G_{M}$ group

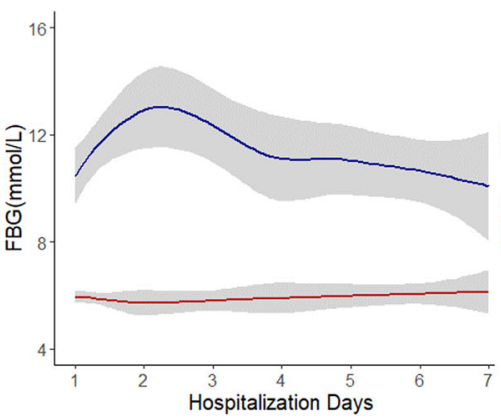

(C) $F B G_{A}$ group

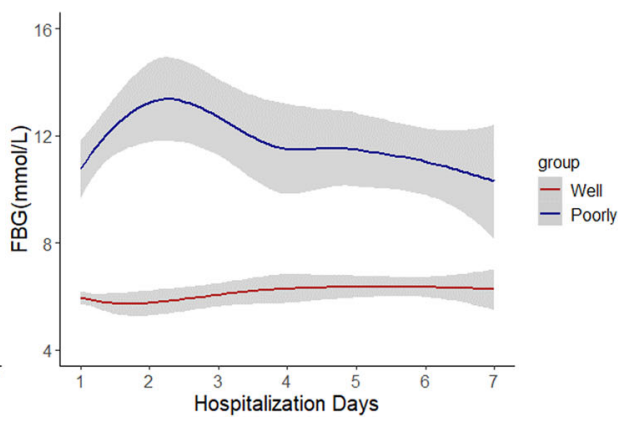

Fig. 1 Dynamic FBG changes in patients of the well controlled and poorly controlled groups defined by $(\mathbf{A}) \mathrm{FBG}_{1}$, $(\mathbf{B}) \mathrm{FBG}_{\mathrm{M}}$, and $(\mathbf{C}) \mathrm{FBG}_{\mathrm{A}}$ during the first 7 days after admission

The major symptoms on admission were fever (68.3\%), cough (61.5\%), fatigue (45.9\%), and dyspnea (17.6\%), similar to the general patient population $[18,19]$. The main comorbidities were hypertension (65.0\%) and CVD (32.5\%). Lab examination on admission showed most subjects had high C-reaction protein (CRP) level (55.6\%) and lymphopenia (33.5\%). About $75 \%$ of patients (155/206) had available records of glycemic control drug use before admission including alpha-glucosidase inhibitors (36.1\%), insulin (29.7\%), metformin (26.5\%), sulfonylurea (19.4\%), DPP-4 inhibitors (2.6\%), and thiazolidinediones $(1.9 \%) .84 .5 \%$ of inpatients received antivirus treatment. Fig. S1 shows HbA1c level correlated with baseline glucose $\left(\mathrm{FBG}_{1}\right) \quad(\mathrm{PCC}=0.728, \quad P=1.13 \mathrm{E}-05), \quad$ maximum $\quad \mathrm{FBG}$ $\left(\mathrm{FBG}_{\mathrm{M}}\right)(\mathrm{PCC}=0.685, P=1.54 \mathrm{E}-05)$, and arithmetic mean of FBG $\left(\mathrm{FBG}_{\mathrm{A}}\right)(\mathrm{PCC}=0.708, P=5.73 \mathrm{E}-06)$ during the first 7 days after hospital admission. All these correlations were stronger than that between $\mathrm{HbAlc}$ and $\mathrm{FBG}_{\mathrm{M}}(\mathrm{PCC}=0.632$, $P=7.93 \mathrm{E}-05$ ) during 28 days after admission, which indicated FBG in 7 days show better consistency with HbA1c than FBG in 28 days. Given that steroid treatment could increase FBG level of patients [20], Fig. S2 shows the dynamic FBG changes of inpatients during 28 days after admission with or without steroid treatment. In comparison to the group without steroid treatment, patients taking steroid therapy had higher FBG levels during hospitalization.

For glucose management defined by $\mathrm{FBG}_{1}$, body temperature, heart rate, respiratory rate and blood pressure show similarity between the well-controlled and poorly controlled groups. Patients in the well-controlled group reported slightly lower frequencies of fever $(67.2 \%$ vs. $70.0 \%)$ and dyspnea (16\% vs. $20 \%$ ) compared to the poorly controlled group. In addition, the proportion of diagnosed severity in the wellcontrolled group is modestly lower than in the poorly controlled (56.0\% vs. $60.5 \%)$. However, pre-existing hypertension (69.6\% vs. $58.0 \%)$ and CVD (36.8\% vs. $25.9 \%)$ were more frequent in the well-controlled group. Patients with wellcontrolled glucose show significantly lower proportions of leukocytosis ( $8.3 \%$ vs. $23.0 \%)$, increased neutrophil counts
Table 2 Associations between blood glucose control groups (with cutoff at $7.8 \mathrm{mmol} / \mathrm{L}$ ) and prognosis of patients with diabetes and COVID-19

\begin{tabular}{llllll}
\hline $\begin{array}{l}\text { Group } \\
\text { definition }\end{array}$ & Model 1 & & & Model 2 & \\
\cline { 2 - 3 } \cline { 5 - 6 } & HR $(95 \% \mathrm{CI})$ & $P$ value & & HR $(95 \% \mathrm{CI})$ & $P$ value \\
\hline $\mathrm{FBG}_{1}$ & $3.95(1.36-11.47)$ & 0.012 & & $3.69(1.11-12.25)$ & 0.033 \\
$\mathrm{FBG}_{\mathrm{M}}$ & $5.07(1.44-17.86)$ & 0.011 & & $4.96(1.27-19.40)$ & 0.021 \\
$\mathrm{FBG}_{\mathrm{A}}$ & $5.62(1.59-19.83)$ & 0.007 & & $5.55(1.41-21.81)$ & 0.014 \\
\hline
\end{tabular}

Model 1 was adjusted for age, gender, and severity classification on admission

Model 2 was adjusted for age, gender, severity classification on admission, hypertension, CVD, COPD, comorbidities, antivirus drug used, CPT therapy and steroid used

$H R$ hazard ratio, $C I$ confidence interval

( $12.5 \%$ vs. $31.1 \%)$, lymphopenia ( $28.3 \%$ vs. $41.9 \%$ ), elevated urea nitrogen levels (7.0\% vs. $12.9 \%$ ), and elevated CRP levels $(49.1 \%$ vs. $67.1 \%)$ than those with poorly controlled glucose. For $\mathrm{FBG}_{\mathrm{M}}$ and $\mathrm{FBG}_{\mathrm{A}}$, the numbers of patient in the wellcontrolled group decreased with illness progression when compared to the group under $\mathrm{FBG}_{1}$ (125 vs. 108, 125 vs. 113), which hints poor glucose management for patient with diabetes during COVID-19 treatment. The dynamic glucose change after admission of well-controlled and poorly controlled groups under different definitions is shown in Fig. 1A-C.

Table 2 presents the results of the associations between FBG control groups and prognosis of patients with diabetes and COVID-19. Compared to the well-controlled group, the HR of the poorly controlled group was 5.07 (95\% CI, $1.44-17.86 ; P=0.011)$ for $\mathrm{FBG}_{\mathrm{M}}$ and $5.62(95 \% \mathrm{CI}$, $1.59-19.83 ; P=0.007)$ for $\mathrm{FBG}_{\mathrm{A}}$ after adjusting for age, sex, and severity classification (Model 1). The findings were also statistically significant for groups in terms of $\mathrm{FBG}_{\mathrm{M}}$ $(\mathrm{HR}=4.96 ; 95 \% \mathrm{CI}, 1.27-19.40 ; P=0.021)$ and $\mathrm{FBG}_{\mathrm{A}}$ $(\mathrm{HR}=5.55 ; 95 \% \mathrm{CI}, 1.41-21.81 ; P=0.014)$ after further adjusting for hypertension, CVD, COPD, other comorbidities, antivirus drug used, CPT therapy and steroid used 
(Model 2). Poorly controlled $\mathrm{FBG}_{1}$ could predict the bad prognosis of patients with diabetes and COVID-19 both in Model $1(\mathrm{HR}=3.95 ; 95 \% \mathrm{CI}, 1.36-11.57 ; P=0.012)$ and Model 2 (HR $=3.69 ; 95 \% \mathrm{CI}, 1.11-12.25 ; P=0.033)$. In addition, we could not find a significant non-linear effect of $\mathrm{FBG}_{1}$ (P for non-linear: 0.622 ) or $\mathrm{FBG}_{\mathrm{A}}$ ( $P$ for non-linear: 0.257 ) levels on prognosis but we did for $\mathrm{FBG}_{\mathrm{M}}$ ( $P$ for nonlinear: 0.016; Fig. S3) under Model 2. Table S1 shows the relationship between the two redefined groups using a cutoff at $10.0 \mathrm{mmol} / \mathrm{L}$. In comparison with the well-controlled group, the HR of the poorly controlled group defined by $\mathrm{FBG}_{\mathrm{M}}$ was statistically significant in both Model 1 ( HR = 4.17; 95\% CI, 1.47-11.82; $P=0.007)$ and Model $2(\mathrm{HR}=$ $3.55 ; 95 \%$ CI, $1.19-11.20 ; P=0.024)$.

\section{Discussion}

In this retrospective study, we examined the effect of glucose management on the prognosis of COVID-19 inpatients with type 2 diabetes. We used admission $\mathrm{FBG}\left(\mathrm{FBG}_{1}\right)$, arithmetic mean of FBG $\left(\mathrm{FBG}_{\mathrm{A}}\right)$, and maximum FBG $\left(\mathrm{FBG}_{\mathrm{M}}\right)$ as surrogates of dynamic FBG for each patient and considered $7.8 \mathrm{mmol} / \mathrm{L}$ as a criteria of well-controlled blood glucose. Our results show that patients who maintained a proper blood glucose control would have a lower risk of admission to ICU or in-hospital death in comparison with poorly controlled patients. Particularly, baseline FBG could predict the prognosis of patients, which means a more attention needs to be paid to patients with diabetes and COVID-19 having high admission FBG. Doctors should invite endocrinologists and nutritionists to participate in the management of inpatients with diabetes and coronavirus infection whenever possible. These also gave hints on the importance of glucose controlling for COVID-19 outpatient with diabetes.

To our knowledge, few studies have been performed on the association between glucose management and prognosis of inpatients with COVID-19 and diabetes. Recently, Zhu et al. having a similar aim also reported the fatal prognosis of patients with poorly controlled glucose [7]. However, there are some differences between the two studies. First, we focused on blood glucose during first 7 days after admission instead of the whole observation period, which was much more meaningful from the perspective of early prediction and more consistent with $\mathrm{HbA1c}$ measurements. Second, multiple indicator (i.e., $\mathrm{FBG}_{1}, \mathrm{FBG}_{\mathrm{A}}$, and $\mathrm{FBG}_{\mathrm{M}}$ ) rather than only maximum blood glucose were employed to act for dynamic glucose levels. Third, Zhu et al. used $10.0 \mathrm{mmol} / \mathrm{L}$ (i.e., targeting level of $2 \mathrm{~h}$ postprandial glucose in diabetes management) as a boundary of glucose control according to the guidelines for prevention and control of type 2 diabetes in China (2017) and we used $7.8 \mathrm{mmol} / \mathrm{L}$ as a cutoff, which stands for preprandial blood glucose levels of hospitalized patients as based on the recommendation from the American Association of Clinical Endocrinologists and American Diabetes Association [9]. Last, the primary endpoint of our study was admission to ICU or in-hospital death instead of 28-day death. Raoufi et al. collected clinical characteristic of 117 patients with coexistent COVID-19 and diabetes and used hemoglobin A1c (HbA1c) as index of glucose management [21]. However, their analysis is crude (ignoring the survival process and confounders adjustment), and no significant difference was observed in mortality rates between the wellcontrolled and poorly-controlled patients. Li et al. included 132 patients with COVID-19 and diabetes and suggested patients with admission glucose $>11 \mathrm{mmol} / \mathrm{L}$ had an increased risk of death and in-hospital complications [22]. But they did not take survival time of inpatients into consideration and make sensitive analysis on cutoff of defining glucose control. Two other small-scale observational studies concentrated on risk factor for prognosis of COVID-19 patients with diabetes instead of blood glucose control [23, 24].

The link between COVID-19 and diabetes/hyperglycemia may be reciprocal. On the one hand, hyperglycemia may increase viral replication in vivo and suppress the host's anti-viral immune response [25, 26]. Besides, expression of angiotensin-converting enzyme 2 (ACE2) was increased in patients with diabetes treated with ACE inhibitors and angiotensin 2 receptor blockers. Consequently, the high expression of ACE2 accelerated viral entry into cells $[6,27,28]$. On the other hand, the SARSCoV-2 virus hijacks an endocrine pathway that plays a crucial role in metabolism and potentially damages pancreatic $\beta$ cells [29, 30]. Moreover, highly expressed proinflammatory cytokines, activation of the renin-angiotensin system, and lifestyle changes might play crucial roles in developing diabetes during this pandemic [31]. A recent meta-analysis estimated a pooled proportion of $14.4 \%$ for newly diagnosed diabetes in COVID-19 inpatients [32]. In this study, we observed hyperglycemia in 192 COVID-19 patients without diabetes and steroid treatment during hospitalization. This might be attributed to a stress response connected with severe illness or the potential diabetogenic effects of COVID-19. Furthermore, a new study also reported Influenza A virus could induce cytokine storm by increasing glucose metabolism [33], while cytokine storms have been shown to poses a major threat for COVID-19 patients [34, 35]. It might be a combination of all these factors that leads to poor prognosis of patients with diabetes suffering from a SARS-CoV-2 infection. Nevertheless, some of these potential mechanisms are based on other coronaviruses and their clinical relevance remains unclear.

Continuous glucose controlling is crucial for inpatients with diabetes and coronavirus infection. Some studies observed the deterioration state in the patients with poor glycemic control [14, 36]. High-dose insulin therapy has 
been recommended for the treatment of severely or critically ill inpatients with COVID-19 and diabetes [14, 31]. However, in order to prevent the excess risk of severe hypoglycemia during intensive insulin therapy, continuous glucose monitoring should be encouraged. Moreover, potassium balance and fluid balance also deserve attentions in the context of high insulin consumption. As a first-line antidiabetic drug, metformin use might be associated with reduced severity and mortality among diabetic patients hospitalized for severe COVID-19 by reducing the level of proinflammatory signaling and cytokine storm $[24,37,38]$. Nevertheless, doctors should carefully monitor the rare side effects of metformin, including lactic acidosis and acute kidney injury, especially for patients with severe symptoms. As suggested by a consensus, SGLT2 inhibitors are not recommended due to the putative risk of dehydration and diabetic ketoacidosis, while DPP-4 inhibitors are well tolerated and can continue to be used [14].

Although this study generated a significant suggestion of glucose management for COVID-19 patients with type 2 diabetes, several limitations should be addressed. First, due to the retrospective nature of the study and the circumstance of the clinical practice during the pandemic, some data was incomplete or unavailable, which possibly weakens our results. For example, laboratory tests were not fixed, which meant some data points were lost in first 7 days, and subsequently decrease the statistical power for analyzing glucose dynamic changes. Our study had litter control on potential confounders such as weight, race, diet and physical activity. Last, this exploratory study is a single-center study with a small sample size. Thus, the interpretation might be limited by selection bias and less statistical efficacy.

\section{Data availability}

Data may be provided upon request to the corresponding author.

Acknowledgements We thank all patients involved in the study and all medical staff involved in the diagnosis and treatment of patients with COVID-19 in Wuhan.

Author contributions W.D., L.L., X.L., and M.Z. designed study, collected and analyzed data, and wrote paper. L.W., W.W., K.L., Y.L., R.D., W.Y., Z.W., B.H., M.W., T.Z., J.L., Y.L., J.S., C.L., and P.L. prepared clinical and laboratory data. X.X., Y.L., Q.W., and S.W. provided valuable suggestions for study design and data analysis. W. D., Y.L., X.X., and S.L. designed the project, edited paper, and supervised the study. All authors have approved the final version of this paper. W.D. is the guarantor of this work and, as such, had full access to all the data in the study and takes responsibility for the integrity of the data and the accuracy of the data analysis.

Funding This research was supported by grants from National Natural Science Foundation of China (Grant No. 91959113, 81972358, 81903409, 81572893), Key Foundation of Wuhan Huoshenshan
Hospital (Grant No. 2020[18]), Key Research \& Development Program of Jiangsu Province (Grant No. BE2017733, BE2018713), Medical Innovation Project of Logistics Service (Grant No. 18JS005), Basic Research Program of Jiangsu Province (Grant No. BK20180036), Development Program of Medical Big Data Analysis Software of Nanjing Medical University (Grant No. 2019KF0106).

\section{Compliance with ethical standards}

Conflict of interest The authors declare no competing interests.

Ethical approval This study was approved by the Medical Ethical Committee of Wuhan Huoshenshan Hospital.

Informed consent The informed consent was waived by the ethics board of the hospital due to urgency need during the pandemic period.

Publisher's note Springer Nature remains neutral with regard to jurisdictional claims in published maps and institutional affiliations.

\section{References}

1. Z. Wu, J.M. McGoogan, Characteristics of and important lessons from the coronavirus disease 2019 (COVID-19) outbreak in China: summary of a report of 72314 cases from the Chinese Center for Disease Control and Prevention. JAMA 323(13), 1239-1242 (2019).

2. X. Yang, Y. Yu, J. Xu, H. Shu, J. Xia, H. Liu, Y. Wu, L. Zhang, Z. Yu, M. Fang, T. Yu, Y. Wang, S. Pan, X. Zou, S. Yuan, Y. Shang, Clinical course and outcomes of critically ill patients with SARS-CoV-2 pneumonia in Wuhan, China: a single-centered, retrospective, observational study. Lancet Respiratory Med. 8, 475-481 (2020)

3. J.J. Zhang, X. Dong, Y.Y. Cao, Y.D. Yuan, Y.B. Yang, Y.Q. Yan, C.A. Akdis, Y.D. Gao, Clinical characteristics of 140 patients infected with SARS-CoV-2 in Wuhan, China. Allergy 75(7), 1730-1741 (2020).

4. W.-J. Guan, Z.-Y. Ni, Y. Hu, W.-H. Liang, C.-Q. Ou, J.-X. He, L. Liu, H. Shan, C.-L. Lei, D.S.C. Hui, B. Du, L.-J. Li, G. Zeng, K.Y. Yuen, R.-C. Chen, C.-L. Tang, T. Wang, P.-Y. Chen, J. Xiang, S.-Y. Li, J.-L. Wang, Z.-J. Liang, Y.-X. Peng, L. Wei, Y. Liu, Y.H. Hu, P. Peng, J.-M. Wang, J.-Y. Liu, Z. Chen, G. Li, Z.-J. Zheng, S.-Q. Qiu, J. Luo, C.-J. Ye, S.-Y. Zhu, N.-S. Zhong, Clinical Characteristics of Coronavirus Disease 2019 in China. N. Engl. J. Med. 382, 1708-1720 (2020)

5. C. Huang, Y. Wang, X. Li, L. Ren, J. Zhao, Y. Hu, L. Zhang, G. Fan, J. Xu, X. Gu, Z. Cheng, T. Yu, J. Xia, Y. Wei, W. Wu, X. Xie, W. Yin, H. Li, M. Liu, Y. Xiao, H. Gao, L. Guo, J. Xie, G. Wang, R. Jiang, Z. Gao, Q. Jin, J. Wang, B. Cao, Clinical features of patients infected with 2019 novel coronavirus in Wuhan, China. Lancet 395, 497-506 (2020)

6. L. Fang, G. Karakiulakis, M. Roth, Are patients with hypertension and diabetes mellitus at increased risk for COVID-19 infection? The Lancet. Respiratory Med. 8, e21 (2020)

7. L. Zhu, Z.G. She, X. Cheng, J.J. Qin, X.J. Zhang, J. Cai, F. Lei, H. Wang, J. Xie, W. Wang, H. Li, P. Zhang, X. Song, X. Chen, M. Xiang, C. Zhang, L. Bai, D. Xiang, M.M. Chen, Y. Liu, Y. Yan, M. Liu, W. Mao, J. Zou, L. Liu, G. Chen, P. Luo, B. Xiao, C. Zhang, Z. Zhang, Z. Lu, J. Wang, H. Lu, X. Xia, D. Wang, X. Liao, G. Peng, P. Ye, J. Yang, Y. Yuan, X. Huang, J. Guo, B.H. Zhang, H. Li, Association of blood glucose control and outcomes in patients with COVID-19 and pre-existing type 2 diabetes. Cell Metab. 31, 1068-1077 e1063 (2020) 
8. A. Extance, Covid-19 and long term conditions: what if you have cancer, diabetes, or chronic kidney disease? Bmj 368, m1174 (2020)

9. E.S. Moghissi, M.T. Korytkowski, M. DiNardo, D. Einhorn, R. Hellman, I.B. Hirsch, S.E. Inzucchi, F. Ismail-Beigi, M.S. Kirkman, G.E. Umpierrez, American Association of Clinical E, American Diabetes A: American Association of Clinical Endocrinologists and American Diabetes Association consensus statement on inpatient glycemic control. Diabetes Care 32, 1119-1131 (2009)

10. S. Wang, P. Ma, S. Zhang, S. Song, Z. Wang, Y. Ma, J. Xu, F. Wu, L. Duan, Z. Yin, H. Luo, N. Xiong, M. Xu, T. Zeng, Y. Jin, Fasting blood glucose at admission is an independent predictor for 28-day mortality in patients with COVID-19 without previous diagnosis of diabetes: a multi-centre retrospective study. Diabetologia 63, 2102-2111 (2020)

11. C. Sardu, N. D’Onofrio, M.L. Balestrieri, M. Barbieri, M.R. Rizzo, V. Messina, P. Maggi, N. Coppola, G. Paolisso, R. Marfella, Outcomes in patients with hyperglycemia affected by COVID-19: can we do more on Glycemic Control? Diabetes Care 43, 1408-1415 (2020)

12. S.P. Liu, Q. Zhang, W. Wang, M. Zhang, C. Liu, X. Xiao, Z. Liu, W. $\mathrm{M}$. Hu, P. Jin, Hyperglycemia is a strong predictor of poor prognosis in COVID-19. Diabetes Res. Clin. Pract. 167, 108338 (2020)

13. J. Zhou, J. Tan, Diabetes patients with COVID-19 need better blood glucose management in Wuhan, China. Metab.: Clin. Exp. 107, 154216 (2020)

14. S.R. Bornstein, F. Rubino, K. Khunti, G. Mingrone, D. Hopkins, A.L. Birkenfeld, B. Boehm, S. Amiel, R.I. Holt, J.S. Skyler, J.H. DeVries, E. Renard, R.H. Eckel, P. Zimmet, K.G. Alberti, J. Vidal, B. Geloneze, J.C. Chan, L. Ji, B. Ludwig, Practical recommendations for the management of diabetes in patients with COVID-19. Lancet Diabetes Endocrinol. 8, 546-550 (2020)

15. A. Ceriello, A.P. Stoian, M. Rizzo, COVID-19 and diabetes management: What should be considered? Diabetes Res. Clin. Pract. 163, 108151 (2020)

16. X. Xia, K. Li, L. Wu, Z. Wang, M. Zhu, B. Huang, J. Li, Z. Wang, W. Wu, M. Wu, W. Li, L. Li, Y. Cai, B. Bosco, A. Zhong, X. Liu, T. Lv, Z. Gan, G. Chen, Y. Pan, C. Liu, K. Zhang, X. Xu, C. Wang, Q. Wang, Improved clinical symptoms and mortality on severe/critical COVID-19 patients utilizing Convalescent Plasma Transfusion. Blood 136(6), 755-759 (2020).

17. P.M. Grambsch, T.M. Therneau, Proportional hazards tests and diagnostics based on weighted residuals. Biometrika 81, 515-526 (1994)

18. N. Chen, M. Zhou, X. Dong, J. Qu, F. Gong, Y. Han, Y. Qiu, J. Wang, Y. Liu, Y. Wei, J. Xia, T. Yu, X. Zhang, L. Zhang, Epidemiological and clinical characteristics of 99 cases of 2019 novel coronavirus pneumonia in Wuhan, China: a descriptive study. Lancet 395, 507-513 (2020)

19. D. Wang, B. Hu, C. Hu, F. Zhu, X. Liu, J. Zhang, B. Wang, H. Xiang, Z. Cheng, Y. Xiong, Y. Zhao, Y. Li, X. Wang, Z. Peng, Clinical characteristics of 138 hospitalized patients with 2019 Novel Coronavirus-Infected Pneumonia in Wuhan, China. JAMA 323(11), 1061-1069 (2020).

20. J.K. Yang, Y. Feng, M.Y. Yuan, S.Y. Yuan, H.J. Fu, B.Y. Wu, G. Z. Sun, G.R. Yang, X.L. Zhang, L. Wang, X. Xu, X.P. Xu, J.C. Chan, Plasma glucose levels and diabetes are independent predictors for mortality and morbidity in patients with SARS. Diabet. Med.: J. Br. Diabet. Assoc. 23, 623-628 (2006)

21. M. Raoufi, S. Khalili, M. Mansouri, A. Mahdavi, N. Khalili, Wellcontrolled vs poorly-controlled diabetes in patients with COVID19: are there any differences in outcomes and imaging findings? Diabetes Res. Clin. Pract. 166, 108286 (2020)

22. Y. Li, X. Han, O. Alwalid, Y. Cui, Y. Cao, J. Liu, J. Gu, L. Wang, Y. Fan, H. Shi, Baseline characteristics and risk factors for short-term outcomes in 132 COVID-19 patients with diabetes in Wuhan China: a retrospective study. Diabetes Res. Clin. Pract. 166, 108299 (2020)

23. Q. Shi, X. Zhang, F. Jiang, X. Zhang, N. Hu, C. Bimu, J. Feng, S. Yan, Y. Guan, D. Xu, G. He, C. Chen, X. Xiong, L. Liu, H. Li, J. Tao, Z. Peng, W. Wang, Clinical characteristics and risk factors for mortality of COVID-19 patients with diabetes in Wuhan, China: A Two-Center, Retrospective Study. Diabetes Care 43, 1382-1391 (2020)

24. Y. Chen, D. Yang, B. Cheng, J. Chen, A. Peng, C. Yang, C. Liu, M. Xiong, A. Deng, Y. Zhang, L. Zheng, K. Huang, Clinical characteristics and outcomes of patients with diabetes and COVID-19 in Association With Glucose-Lowering Medication. Diabetes Care 43, 1399-1407 (2020)

25. M.E. Morra, L. Van Thanh, M.G. Kamel, A.A. Ghazy, A.M.A. Altibi, L.M. Dat, T.N.X. Thy, N.L. Vuong, M.R. Mostafa, S.I. Ahmed, S.S. Elabd, S. Fathima, T. Le Huy Vu, A.S. Omrani, Z.A. Memish, K. Hirayama, N.T. Huy, Clinical outcomes of current medical approaches for Middle East respiratory syndrome: a systematic review and meta-analysis. Rev. Med. Virol. 28, e1977 (2018)

26. M.A. Hill, C. Mantzoros, J.R. Sowers, Commentary: COVID-19 in patients with diabetes. Metab.: Clin. Exp. 107, 154217 (2020)

27. P. Zhang, L. Zhu, J. Cai, F. Lei, J.J. Qin, J. Xie, Y.M. Liu, Y.C. Zhao, X. Huang, L. Lin, M. Xia, M.M. Chen, X. Cheng, X. Zhang, D. Guo, Y. Peng, Y.X. Ji, J. Chen, Z.G. She, Y. Wang, Q. Xu, R. Tan, H. Wang, J. Lin, P. Luo, S. Fu, H. Cai, P. Ye, B. Xiao, W. Mao, L. Liu, Y. Yan, M. Liu, M. Chen, X.J. Zhang, X. Wang, R. M. Touyz, J. Xia, B.H. Zhang, X. Huang, Y. Yuan, L. Rohit, P.P. Liu, H. Li, Association of inpatient use of Angiotensin-Converting Enzyme inhibitors and Angiotensin II Receptor Blockers With mortality among patients with hypertension Hospitalized with COVID-19. Circulation Res. 126, 1671-1681 (2020)

28. R. Pal, A. Bhansali, COVID-19, diabetes mellitus and ACE2: The conundrum. Diabetes Res. Clin. Pract. 162, 108132 (2020)

29. M. Hoffmann, H. Kleine-Weber, S. Schroeder, N. Kruger, T. Herrler, S. Erichsen, T.S. Schiergens, G. Herrler, N.H. Wu, A. Nitsche, M.A. Muller, C. Drosten, S. Pohlmann, SARS-CoV-2 cell entry depends on ACE2 and TMPRSS2 and is blocked by a clinically proven protease inhibitor. Cell 181, 271-280 e278 (2020)

30. F. Rubino, S.A. Amiel, P. Zimmet, G. Alberti, S. Bornstein, R.H. Eckel, G. Mingrone, B. Boehm, M.E. Cooper, Z. Chai, S. Del Prato, L. Ji, D. Hopkins, W.H. Herman, K. Khunti, J.C. Mbanya, E. Renard, New-onset diabetes in Covid-19. N. Engl. J. Med. 383 (8), 789-790 (2020).

31. T. Sathish, R.J. Tapp, M.E. Cooper, P. Zimmet, Potential metabolic and inflammatory pathways between COVID-19 and newonset diabetes. Diabetes Metab. 47, 101204 (2021)

32. T. Sathish, N. Kapoor, Y. Cao, R.J. Tapp, P. Zimmet, Proportion of newly diagnosed diabetes in COVID-19 patients: a systematic review and meta-analysis. Diabetes Obes. Metab. 23, 870-874 (2021)

33. Q. Wang, P. Fang, R. He, M. Li, H. Yu, L. Zhou, Y. Yi, F. Wang, Y. Rong, Y. Zhang, A. Chen, N. Peng, Y. Lin, M. Lu, Y. Zhu, G. Peng, L. Rao, S. Liu, O-GlcNAc transferase promotes influenza A virus-induced cytokine storm by targeting interferon regulatory factor-5. Sci. Adv. 6, eaaz7086 (2020)

34. M. Catanzaro, F. Fagiani, M. Racchi, E. Corsini, S. Govoni, C. Lanni, Immune response in COVID-19: addressing a pharmacological challenge by targeting pathways triggered by SARS-CoV2. Signal Transduct. Target. Ther. 5, 84 (2020)

35. M.F. Konig, M. Powell, V. Staedtke, R.Y. Bai, D.L. Thomas, N. Fischer, S. Huq, A.M. Khalafallah, A.Koenecke, R.Xiong, B. Mensh, N.Papadopoulos, K.W.Kinzler, B.Vogelstein, J.T.Vogelstein, S.Athey, S.Zhou, C.Bettegowda, Preventing cytokine storm syndrome in COVID-19 using alpha-1 adrenergic receptor antagonists. J. Clin. Invest. 130(7), 3345-3347 (2020). 
36. A.J. Scheen, M. Marre, C. Thivolet, Prognostic factors in patients with diabetes hospitalized for COVID-19: Findings from the CORONADO study and other recent reports. Diabetes Metab. 46, 265-271 (2020)

37. K. Khunti, P. Knighton, F. Zaccardi, C. Bakhai, E. Barron, N. Holman, P. Kar, C. Meace, N. Sattar, S. Sharp, N.J. Wareham, A. Weaver, E. Woch, B. Young, J. Valabhji, Prescription of glucose- lowering therapies and risk of COVID-19 mortality in people with type 2 diabetes: a nationwide observational study in England. Lancet Diabetes Endocrinol. 9, 293-303 (2021)

38. O. Kamyshnyi, V. Matskevych, T. Lenchuk, O. Strilbytska, K. Storey, O. Lushchak, Metformin to decrease COVID-19 severity and mortality: Molecular mechanisms and therapeutic potential. Biomedicine Pharmacother. 144, 112230 (2021) 